\title{
Erratum to: Endoplasmic reticulum (ER) stress and the unfolded protein response (UPR) in plants
}

\author{
Shucen Wan ${ }^{1,3} \cdot$ Liwen Jiang ${ }^{2,3}$
}

Published online: 2 July 2015

(C) Springer-Verlag Wien 2015

\section{Erratum to: Protoplasma}

DOI 10.1007/s00709-015-0842-1

The author wishes to correct the following mistakes:

1. Page 3, paragraph 1 in the left column: all the phrase "bZIP17/28" should be "bZIP28".

2. Page 4, Fig. 2: all the phrase "bZIP17/28" in this figure and its caption should be "bZIP28"

3. Page 5, paragraph 1 in the right column, the last sentence: "but only AtIRE1b is responsible for bZIP60 mRNA splicing" should be "but $A t \mathrm{IRE} 1 \mathrm{~b}$ may carry more responsibility for bZIP60 mRNA splicing"

4. Page 8, paragraph 4 in the left column, line 10: "important" should be "impertinent"

5. Page 9, paragraph 1 in the left column, line 5: "In plants" should be "In animals"

The online version of the original article can be found at http://dx.org.doi/ 10.1007/s00709-015-0842-1.

Shucen Wan

sarabiwan@gmail.com

$\triangle$ Liwen Jiang

ljiang@cuhk.edu.hk

1 Molecular Biotechnology Program, School of Life Sciences, The Chinese University of Hong Kong, Shatin, New Territories, Hong Kong, China

2 Centre for Cell and Developmental Biology and State Key Laboratory of Agrobiotechnology, School of Life Sciences, The Chinese University of Hong Kong, Shatin, New Territories, Hong Kong, China

3 School of Life Sciences, The Chinese University of Hong Kong, Shatin, New Territories, Hong Kong, China 